\title{
Bayesian Approach for Sequential Probabilistic Back Analysis of Uncertain Geomechanical Parameters and Reliability Updating of Tunneling-Induced Ground Settlements
}

\author{
Cong Li, ${ }^{1,2}$ Shui-Hua Jiang $\mathbb{D}^{1,}{ }^{1,3}{\text { Jinhui } \mathrm{Li}^{4},{ }^{4} \text { and Jinsong Huang }}^{1}$ \\ ${ }^{1}$ School of Civil Engineering and Architecture, Nanchang University, 999 Xuefu Road, Nanchang 330031, China \\ ${ }^{2}$ School of Civil Engineering and Architecture, Wuhan Polytechnic University, Wuhan 430023, Hubei Province, China \\ ${ }^{3}$ State Key Laboratory of Geomechanics and Geotechnical Engineering, Institute of Rock and Soil Mechanics, \\ Chinese Academy of Sciences, Wuhan 430071, China \\ ${ }^{4}$ Department of Civil and Environmental Engineering, Harbin Institute of Technology (Shenzhen), Shenzhen 518055, China \\ Correspondence should be addressed to Shui-Hua Jiang; sjiangaa@ncu.edu.cn
}

Received 1 September 2019; Revised 20 May 2020; Accepted 25 May 2020; Published 24 June 2020

Academic Editor: Paolo Castaldo

Copyright (C) 2020 Cong Li et al. This is an open access article distributed under the Creative Commons Attribution License, which permits unrestricted use, distribution, and reproduction in any medium, provided the original work is properly cited.

\begin{abstract}
This paper proposes a new sequential probabilistic back analysis approach for probabilistically determining the uncertain geomechanical parameters of shield tunnels by using time-series monitoring data. The approach is proposed based on the recently developed Bayesian updating with subset simulation. Within the framework of the proposed approach, a complex Bayesian back analysis problem is transformed into an equivalent structural reliability problem based on subset simulation. Hermite polynomial chaos expansion-based surrogate models are constructed to improve the computational efficiency of probabilistic back analysis. The reliability of tunneling-induced ground settlements is updated in the process of sequential back analyses. A real shield tunnel project of No. 1 Nanchang Metro Line in China is investigated to assess the effectiveness of the approach. The proposed approach is able to infer the posterior distributions of uncertain geomechanical parameters (i.e., Young's moduli of surrounding soil layers and ground vehicle load). The reliability of tunneling-induced ground settlements can be updated in a real-time manner by fully utilizing the time-series monitoring data. The results show good agreement with the variation trend of field monitoring data of ground settlement and the post-event investigations.
\end{abstract}

\section{Introduction}

In order to alleviate the pressure of urban traffic, constructing underground rail transit network has become a main development of urban transportation system. Urban metro shield tunnel construction often leads to surrounding ground movements and further endangers adjacent structures, which, in turn, poses great threat to the tunnel itself (e.g., $[1,2])$. To mitigate the adverse impact of shield tunnel construction on the surrounding environment, the geomechanical parameters that can reflect the performance of a specific construction site and help to analyze the shield tunneling-induced ground subsidence shall be rationally determined (e.g., $[3,4])$. Typically, only limited amount of field observation data (including geological survey data, test data, and monitoring data) can be acquired for a specific construction site due to the restrictions on the construction cost, time, and project sites (e.g., $[5,6])$. Therefore, how to obtain the geomechanical parameters of the shield tunnels with low uncertainty efficiently based on the limited field observation data remains an unsolved problem.

To make up for the deficiency of field observation data, many researches estimated the geomechanical parameters of shield tunnels through back analysis. For example, Chi et al. [7] applied an optimization technique for the back analysis of tunneling-induced ground movement based on monitoring data. Zhu et al. [8] proposed an artificial bee colony algorithm-based displacement back analysis method to determine the subsoil parameters during shield tunneling process. Gao and Ge [4] employed evolutionary neural 
networks to back analyze the soil parameters and the initial geo-stress of Longtan tunnel and evaluate the stability of the tunnel. Although significant advances have been made in the back analysis of shield tunnel parameters, the above-mentioned studies do not account for the influence of the inherent variability of geomechanical parameters (e.g., $[4,9])$. It is widely accepted that the geomechanical parameters vary spatially and temporally because of the concealment of underground space and complexity of underground environment (e.g., [10-16]). To obtain the shield tunnel parameters in line with the engineering practice, the uncertainties of the geomechanical parameters should be taken into account in the back analysis.

Several studies have investigated probabilistic back analysis problems of uncertain parameters for the shield tunnels by combining probability theory and statistics. For example, Haas and Einstein [17] employed a Markov chain Monte Carlo (MCMC) method to update the posterior distributions of surrounding rock mass parameters of tunnels based on the monitoring data. Špačková and Straub [18] proposed a dynamic Bayesian network based-tunneling process model to update the probability of tunnel failure by utilizing the observation data from geological survey and construction stages. Park and Park [19] conducted a probabilistic back analysis of uncertain parameters for tunnel surrounding rock masses by adopting a response surface method. Liu et al. [20] also employed the MCMC method for the probabilistic back analysis of subsoil cohesion and internal friction angle around a tunnel below river. Although many investigations on the probabilistic back analysis of shield tunnel parameters have been carried out, the time-series monitoring data that are collected during the tunnel advancement were rarely incorporated in the back analysis. To the best of our knowledge, only Miro et al. [21] applied a MCMC method in the probabilistic back analysis of uncertain parameters for surrounding rock masses by utilizing the tunnel-induced ground movements in a sequential manner. However, the MCMC method adopted by Miro et al. [21] is not effective for high dimensional problems, and the reliability of shield tunnels with the probabilistic back analysis results was not investigated. The updated statistics (i.e., means, standard deviations, probability distributions) of geomechanical parameters via the probabilistic back analyses can provide an important basis for reliability analysis of shield tunnels. Based on the updated probability distributions of the uncertain parameters, more realistic reliability assessment of the tunnel face stability or serviceability limit state can be achieved.

In this paper, a Bayesian Updating with Subset simulation (BUS)-based sequential probabilistic back analysis approach is proposed for estimating uncertain geomechanical parameters and updating the reliability of tunneling-induced ground settlements. To improve computational efficiency, the proposed approach transforms a complex Bayesian back analysis problem into an equivalent structural reliability problem which is then solved by subset simulation. The Hermite polynomial chaos expansion-based surrogate models are constructed. In Section 2, the proposed approach comprising the construction of surrogate models and likelihood functions, inference of posterior distribution using BUS approach, and reliability updating of tunnelinginduced ground settlements is presented. In Section 3, the No. 1 Nanchang Metro Line tunnel is treated as a typical case for probabilistically determining the uncertain geomechanical parameters based on the time-series monitoring data of ground settlement. Finally, the reliability of tunneling-induced ground settlements is updated on the basis of the probabilistic back analysis results.

\section{BUS-Based Sequential Probabilistic Back Analysis Approach}

Shield tunneling is often fraught with inevitable uncertainties that include, but not limited to, (1) the inherent variability of soils, (2) the measurement errors in quantifying the soil properties by field and laboratory tests, and (3) the limited knowledge on geotechnical conditions and simplified geotechnical models in predicting the ground settlements (e.g., $[1,21])$. These uncertainties can be reduced by incorporating the field observation data into a Bayesian back analysis by estimating the posterior joint probability density function (PDF) of geomechanical parameters (e.g., $[9,22]$ ). In this study, a BUS-based sequential probabilistic back analysis approach is proposed to estimate the posterior PDF, and its implementation procedure is briefly introduced as follows:

(1) Determine the prior statistics (i.e., mean, coefficient of variation (COV), and distribution) based on the knowledge (e.g., geological survey reports, engineering experience, and existing data in the literature) about the uncertain input parameters.

(2) Construct Hermite polynomial chaos expansion (HPCE)-based surrogate models to facilitate the calculation of tunneling-induced ground settlements for each excavation step.

(3) Establish the likelihood function using the monitoring data of ground settlement obtained from the first excavation step.

(4) Infer the posterior PDFs of uncertain input parameters using the BUS approach. Then, treat the posterior PDFs as the prior PDFs, and build a new likelihood function using the monitoring data of ground settlement obtained from the next excavation step until the last regarded excavation stage.

(5) Update the reliability of tunneling-induced ground settlements for each excavation step based on the obtained posterior statistics of uncertain input parameters.

The flowchart of the BUS-based sequential probabilistic back analysis approach is presented in Figure 1. The involved construction of surrogate models and likelihood functions, inference of posterior distribution using the BUS approach, and reliability updating of tunneling-induced ground settlements are presented in the following subsections. 
Framework of sequential probabilistic back analysis and reliability updating

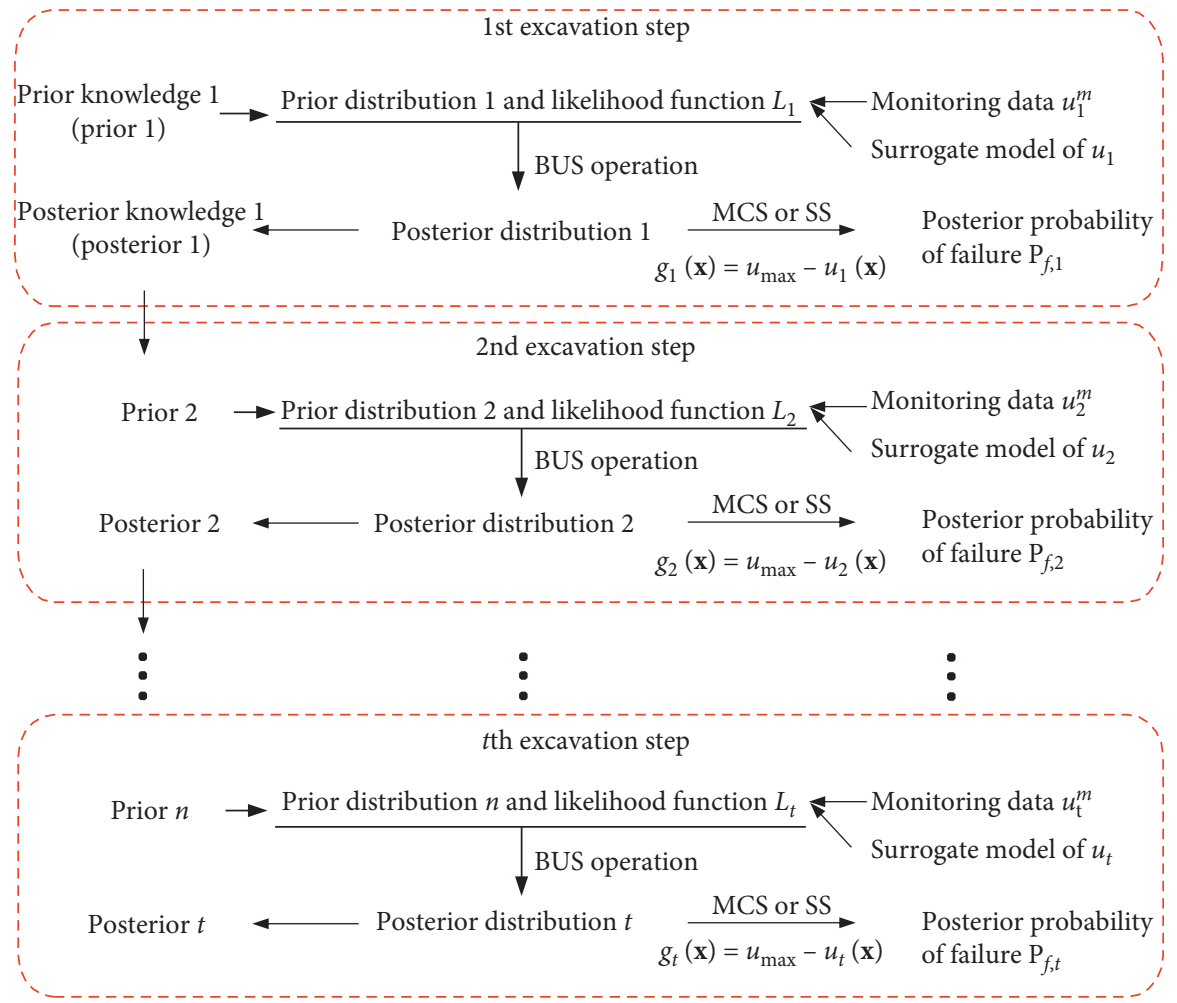

FIGURE 1: Flowchart of BUS-based sequential probabilistic back analysis and reliability updating approach.

2.1. Construction of Surrogate Models and Likelihood Functions. The ground settlements due to shield tunneling and the corresponding limit state functions are commonly the nonlinear implicit functions of the geomechanical parameters, which need to be evaluated through deterministic finite element or finite difference analyses. Generally, tens of thousands of finite element or finite difference analyses are required in a Bayesian back analysis [23], which is computationally demanding for a complicated shield tunnel model. To avoid the high computational burden in the probabilistic back analysis, the polynomial chaos expansion or multivariate adaptive regression splines-based response surface method can be adopted to construct the surrogate models of the ground settlements (e.g., [24, 25]). The response surface method can provide a powerful tool to approximate the nonlinear implicit limit state functions and has many successful applications (e.g., [26, 27]). This study applies the HPCE to construct the surrogates of deterministic numerical models for facilitating the calculation of ground settlements for each excavation step. A surrogate model of the ground settlement $u_{j}$ at the $j^{\text {th }}$ excavation step that involves the uncertain input parameters can be constructed as follows (e.g., $[11,28,29])$ :

$$
\begin{aligned}
u_{j}= & a_{0} \Gamma_{0}+\sum_{i_{1}=1}^{n} a_{i_{1}} \Gamma_{1}\left(\xi_{i_{1}}\right)+\sum_{i_{1}=1}^{n} \sum_{i_{2}=1}^{i_{1}} a_{i_{1} i_{2}} \Gamma_{2}\left(\xi_{i_{1}}, \xi_{i_{2}}\right) \\
& +\sum_{i_{1}=1}^{n} \sum_{i_{2}=1}^{i_{1}} \sum_{i_{3}=1}^{i_{2}} a_{i_{1} i_{2} i_{3}} \Gamma_{3}\left(\xi_{i_{1}}, \xi_{i_{2}}, \xi_{i_{3}}\right)+\cdots
\end{aligned}
$$

where $j=1,2, \ldots, t, t$ is the total number of excavation steps; $n$ is the number of random variables; $a_{0}, a_{i_{1}}, a_{i_{1}, i_{2}}, a_{i_{1}, i_{2}, i_{3}}, \ldots$ are the unknown coefficients; $\Gamma_{j_{p}}(\cdot), j_{p}=1,2,3, \ldots$ are Hermit polynomials with $j_{p}$ degrees of freedom [30]; and $\xi=\left(\xi_{1}, \xi_{2}, \ldots, \xi_{n}\right)^{T}$ are a set of independent standard normal random variables. For the $n_{\mathrm{HPCE}}$-th order HPCE, there are a total of $M=\left(n+n_{\mathrm{HPCE}}\right) ! /\left(n ! \times n_{\mathrm{HPCE}} !\right)$ unknown coefficients (i.e., $\left.a_{0}, a_{i_{1}}, a_{i_{1}, i_{2}}, a_{i_{1}, i_{2}, i_{3}}, \ldots\right)$ in equation (1). The unknown coefficients in the HPCE can be evaluated by solving a series of linear equations given by equation (1). The left side of the linear equations is $u_{j}$ evaluated via deterministic finite element or finite difference analysis based on $N$ realizations of the random variables that are generated by Latin Hypercube Sampling (LHS) technique (e.g., [31], in which $N \geq M$ ).

Having obtained the explicit and approximate expressions for calculating the ground settlements at different shield excavation steps in equation (1), the likelihood functions with the consideration of the uncertainties can be constructed. The likelihood function reflects the model fit with the field observation data for given input parameters $\mathbf{X}=\left(X_{1}, X_{2}, \ldots, X_{n}\right)^{\mathrm{T}}$. Typically, the difference (i.e., $\varepsilon_{m_{j}}$ measurement error) between a measured and a simulated ground settlement for the given values $\mathbf{x}$ of random variables $\mathbf{X}$ can be expressed as

$$
\varepsilon_{m_{j}}=u_{j}^{m}-u_{j}(\mathbf{x})
$$

where $u_{j}^{m}$ is the measurement of the ground settlement which is made at the $j^{\text {th }}$ excavation step and $u_{j}(\mathbf{x})$ is 
evaluated through the deterministic analysis. Note that a mathematical transformation between $\mathbf{x}$ and $\xi$ in equation (1) can be done by a Nataf transformation procedure [29]. Following Miro et al. [21], $\varepsilon_{m_{j}}, j=1,2, \ldots, t$, are assumed to be independent and obey normal distributions with zero mean and constant standard deviations of $\sigma_{\varepsilon_{m}}$. Based on these, the likelihood functions at the $j^{\text {th }}$ excavation step can be established as follows [32]:

$$
L_{j}(\mathbf{x})=\frac{\phi\left\{\left[u_{j}^{m}-u_{j}(\mathbf{x})\right] / \sigma_{\varepsilon_{m_{j}}}\right\}}{\sigma_{\varepsilon_{m_{j}}}},
$$

where $\phi(\cdot)$ is the PDF of a standard normal variable. With the constructed surrogate models using equation (1), the computational cost taken on the evaluations of likelihood functions can be substantially reduced, and so are the total computational costs of the sequential probabilistic back analysis.

2.2. Inference of Posterior Distribution. BUS approach is adopted herein to infer the posterior distributions of the uncertain input parameters, which defines the Bayesian back analysis problem as an equivalent structural reliability problem [23]. Subset simulation (SS) is then employed to solve the structural reliability problem to obtain samples from $f_{\mathbf{X}}^{\prime \prime}(\mathbf{x})$ (e.g., $[33,34])$. The field observation information collected at the $j^{\text {th }}$ excavation step can be described by a likelihood function $L_{j}(\mathbf{x})$ which is utilized to define a failure domain $\Omega_{\mathbf{X}}$ in an augmented outcome space $\mathbf{x}_{+}=[\mathbf{x} ; p]$ :

$$
\Omega_{\mathbf{X}}=\left\{H_{j}\left(\mathbf{x}_{+}\right) \leq 0\right\},
$$

where $H_{j}\left(\mathbf{x}_{+}\right)$is the limit state function which is given by [35]

$$
H_{j}\left(\mathbf{x}_{+}\right)=\ln p-\ln \left[c L_{j}(\mathbf{x})\right]
$$

where $p$ is the realization of a standard uniform random variable in $[0,1]$ that is independent with $\mathbf{x}$ and $c$ is a likelihood multiplier that satisfies the following inequality for all $\mathbf{x}$ [23]:

$$
c L(\mathbf{x}) \leq 1.0 .
$$

It can be noted that sampling the posterior distribution of $\mathbf{x}$ is equivalent to finding the samples generated from the prior distribution of $\mathbf{X}$ and falling in the domain $\Omega_{\mathbf{X}}$ when determining the probability of information event $Z, P(Z)$ $[36,37]$. As the subset simulation does, the BUS approach can also express the $P(Z)$ as a product of larger conditional probabilities of a series of nested intermediate events:

$$
P(Z)=P\left[H_{j}\left(\mathbf{x}_{+}\right) \leq 0\right]=P\left(Z_{1}\right) \prod_{i=2}^{m} \operatorname{Pr}\left(Z_{i} \mid Z_{i-1}\right),
$$

where $Z_{1}^{Z_{2} \ldots Z_{m-1}^{Z_{m}}}$ are intermediate events defined as $Z_{i}=$ $\left\{H\left(\mathbf{x}_{+}\right)<g_{i}\right\}$, in which $g_{i}, i=1,2, \ldots, m$, are threshold values satisfying $g_{1}>g_{2}>\ldots>g_{m-1}>0 \geq g_{m} ; m$ is the number of subset levels required to reach the domain $\Omega_{\mathbf{X}} ; P\left(Z_{1}\right)$ is the probability of the first subset level; and $P\left(Z_{i} \mid Z_{i-1}\right)$ is the conditional probability of $Z_{i}$ given $Z_{i-1}$. Specifically, a modified Metropolis algorithm proposed by Au and Beck [33] is adopted for the computation of the conditional probabilities of $P\left(Z_{i} \mid Z_{i-1}\right), i=2,3, \ldots, m$. The threshold values $g_{i}, i=1,2, \ldots$, $m$, are determined adaptively such that the intermediate conditional probabilities take a target value $p_{0}$.

Once the failure region $\Omega_{\mathrm{X}}$ is reached, the failure samples $\mathbf{x}_{f}$ in the final subset level are extracted and utilized to estimate the posterior statistics of the uncertain input parameters and compute $P(Z)$. The computational effort of the BUS approach decreases significantly with the logarithm of $P(Z)$, which in turn is proportional to the value of the constant $c$. To this end, the value of $c$ is usually selected as large as possible such that equation (6) holds. Following Betz et al. [35] and Jiang et al. [36, 37], $c$ is adaptively estimated as the reciprocal of the maximum of the likelihood function values over the samples at the current subset level, i.e.,

$$
\begin{array}{r}
c_{i}=\frac{1}{\max \left[c_{i-1}^{-1},\left\{L\left(\mathbf{x}_{i, k}\right), k=1,2, \ldots, N_{l}\right\}\right]}, \\
(i=1,2, \ldots, m),
\end{array}
$$

where $N_{l}$ is the number of samples at each subset level; $c_{1} \geq c_{2} \geq \ldots \geq c_{m}$ used in different subset levels should guarantee that the intermediate failure domain $Z_{i}$ is entirely contained in the $Z_{i-1}, i=2,3, \ldots, m$.

2.3. Reliability Updating of Tunneling-Induced Ground Settlements. Once the posterior statistics of the uncertain geomechanical parameters are obtained via the probabilistic back analysis, the reliability of tunneling-induced ground settlements for each excavation step can be updated. The limit state function expressing the maximal ground settlement exceeding an admissible threshold can be defined as

$$
g_{j}(\mathbf{x})=u_{\max }-u_{j}(\mathbf{x})
$$

where $u_{\max }$ is the admissible threshold of ground settlement. The posterior probability $\left(P_{f, j}\right)$ of tunneling-induced ground collapse can be estimated using direct Monte Carlo simulation (MCS) as follows:

$$
P_{f, j}=P\left(F_{j} \mid Z\right)=\frac{P\left(F_{j} \cap Z\right)}{P(Z)}=\frac{\sum_{k=1}^{N_{f}} I\left[u_{\max } \leq u_{j}\left(\mathbf{x}_{f}^{k}\right)\right]}{N_{f}},
$$

where $F$ denotes the ground collapse event, $F_{j}=\left\{\mathbf{x} \in \Omega_{F_{j}}\right\}$, in which $\Omega_{F_{j}}=\left\{g_{j}(\mathbf{x}) \leq 0\right\} ; N_{f}$ is the number of posterior samples $\mathbf{x}_{f}$; and $I(\cdot)$ is the indicator function. In most cases, the joint probability $P\left(F_{j} \cap Z\right)$ is very small; thus the estimate of $P_{f, j}$ using the direct MCS will become rather time consuming. Alternatively, Jiang et al. [36] proposed to conduct a new SS operation following the BUS operation for calculating $P_{f, j}$. Interested readers can refer to Jiang et al. [36] for detailed procedures for estimating $P_{f, j}$. 


\section{Project Background}

3.1. Shield Tunnel Overview. The shield tunnel project of No. 1 Nanchang Metro Line is located in Jiangxi province, China. It is a single-line and double-tunnel structure and about $28 \mathrm{~km}$ in length. The shield tunneling interval originates from the Aixi lake west station, passes through the front square of China telecom of Nanchang branch and Beijing east road, and finally arrives at the Gaoxin avenue station. The Beijing east road is the main traffic artery in Nanchang city. The ground traffic volume is large particularly on the holiday. The underground pipelines are complex, and thus the tunneling-induced ground subsidence is relatively easy to occur. The shield tunnel in this interval has a diameter of $D=6.0 \mathrm{~m}$ and a buried depth of $H=14.0 \mathrm{~m}$, which is constructed with a single-circle shield machine. Figure 2 shows the profile of soil layers surrounding the shield tunnel. For the concerned tunnel interval, the design of five excavation steps is listed in Table 1. The total length of shield tunnel excavation is $1.12 \mathrm{~km}$.

During the shield tunnel construction from the Gaoxin avenue station to the Aixi lake west station, a ground collapse accident occurred at the $827^{\text {th }}$ ring on October 2, 2012. Post-event investigations of this accident found the leakage of the underground water pipes in the silty clay layer and the variation of subsoil property caused by the shield excavation disturbance were the main reasons of the collapse of the $827^{\text {th }}$ ring. The Beijing east road above the shield tunnel was the main traffic artery, so the increase in the vehicle loads during the Chinese National day in 2012 was another main cause. In addition, the shield tunneling construction intensively occurred in the gravel layer (see Figure 2). The monitoring data of ground settlement obtained from the point Ds826 is utilized for sequential probabilistic back analysis of uncertain geomechanical parameters and reliability updating. Note that the point Ds826 is installed on the $826^{\text {th }}$ ring and close to the collapsed $827^{\text {th }}$ ring. Figure 3 presents the monitoring data of ground settlement collected from the point Ds826 at the five excavation steps.

3.2. Numerical Model and Parameters. The shield tunneling interval between the $821^{\text {th }}$ and $845^{\text {th }}$ rings is selected to establish the numerical model using the finite difference programme $\mathrm{FLAC}^{3 \mathrm{D}}$. Figure 4 illustrates a three-dimensional (3D) finite difference model of the tunnel, which has a length of $42 \mathrm{~m}$ in the $Y$-axis direction, a width of 30 in the $X$ axis direction, and a depth of $35 \mathrm{~m}$ in the $Z$-axis direction. With regard to the boundary conditions, the normal movements on the all sides of the 3D model are restrained, whereas the bottom of the model is not allowed to move in the three directions. The excavation face of the model is free, but the nodes around the excavated tunnel have a fixed radial displacement. To simulate the influence of the ground moving vehicle loads on shield tunnel excavation, a uniform vehicle load of $q=10 \mathrm{kPa}$ is applied to the top of the model in accordance to Yang et al. [38].

The commonly used elastic and perfectly plastic model based on the Mohr-Coulomb failure criterion is utilized to represent the stress-strain behavior of the subsoil masses. The initial stress is generated by applying gravitational acceleration to the model. The subsoil masses surrounding the tunnel are modeled using the cylinder elements, while the rest is modeled using the hexahedral elements. The excavated tunnel is lined with a linear elastic material with a Young's modulus of $3.45 \mathrm{GPa}$, a Poisson's ratio of 0.2 , and a density of $2450 \mathrm{~kg} / \mathrm{m}^{3}$. The lining segment is prefabricated with C50 concrete with a thickness of $0.3 \mathrm{~m}$ and a width of $1.2 \mathrm{~m}$, which is modeled using a shell element. A discretization of the model with a total number of 34,400 elements and 37,154 nodes is adopted after a preliminary study of the influence of mesh size. According to Mollon et al. [3], the considered tunnel may result in large ground settlements since it corresponds to a shallow tunnel with the overburden depth being about 2.33 times the outer diameter. For simplicity, the groundwater table is not considered in this study.

The geomechanical parameters of different soil layers are determined based on the geological survey reports [39], as summarized in Table 2. According to the post-event investigations as mentioned in Section 3.1, Young's modulus $E_{1}$ of the gravel layer, Young's modulus $E_{2}$ of the silty clay layer, and ground vehicle load $q$ that are closely related to the ground collapse are identified as random variables after a simple parametric sensitivity study. The prior information of the three random variables (i.e., $E_{1}, E_{2}$, and $q$ ) is determined on the basis of the field observation data and existing data in the literature (e.g., [28, 38, 40-42]). The prior statistics of three random variables are summarized in Table 3.

3.3. Construction and Validation of Surrogate Models. Typically, 3D deterministic finite difference analysis of tunneling-induced ground settlements suffers from excessive computational effort. To improve the computational efficiency of the probabilistic back analysis, the $4^{\text {th }}$ order HPCE-based surrogate models of ground settlements are constructed for different excavation steps in advance. For each excavation step, the number of unknown coefficients of the $4^{\text {th }}$ order HPCE is $M=35 . N=70$ random samples are generated according to the prior statistics of three random variables by the LHS technique to establish the linear equations and determine the unknown coefficients. The expansion terms and the corresponding coefficients of the $4^{\text {th }}$ order HPCE for the $1^{\text {st }}$ excavation step are listed in Table 4.

To balance the computational accuracy and efficiency, 100 direct MCS random samples are generated to verify the surrogate models. Based on these 100 random samples, the probability distributions of the uncertain geomechanical parameters and ground settlements can be inferred with acceptable accuracy. Figures 5(a)-5(f) compare the tunneling-induced ground settlements (i.e., $u_{1}, u_{2}$, and $u_{5}$ ) and their PDFs for three representative excavation steps (i.e., 1, 2, and 5) determined from the $4^{\text {th }}$ order HPCE-based surrogate models and original deterministic finite difference analyses using these 100 random samples, respectively. As observed from Figure 5, the ground settlements and their PDFs 


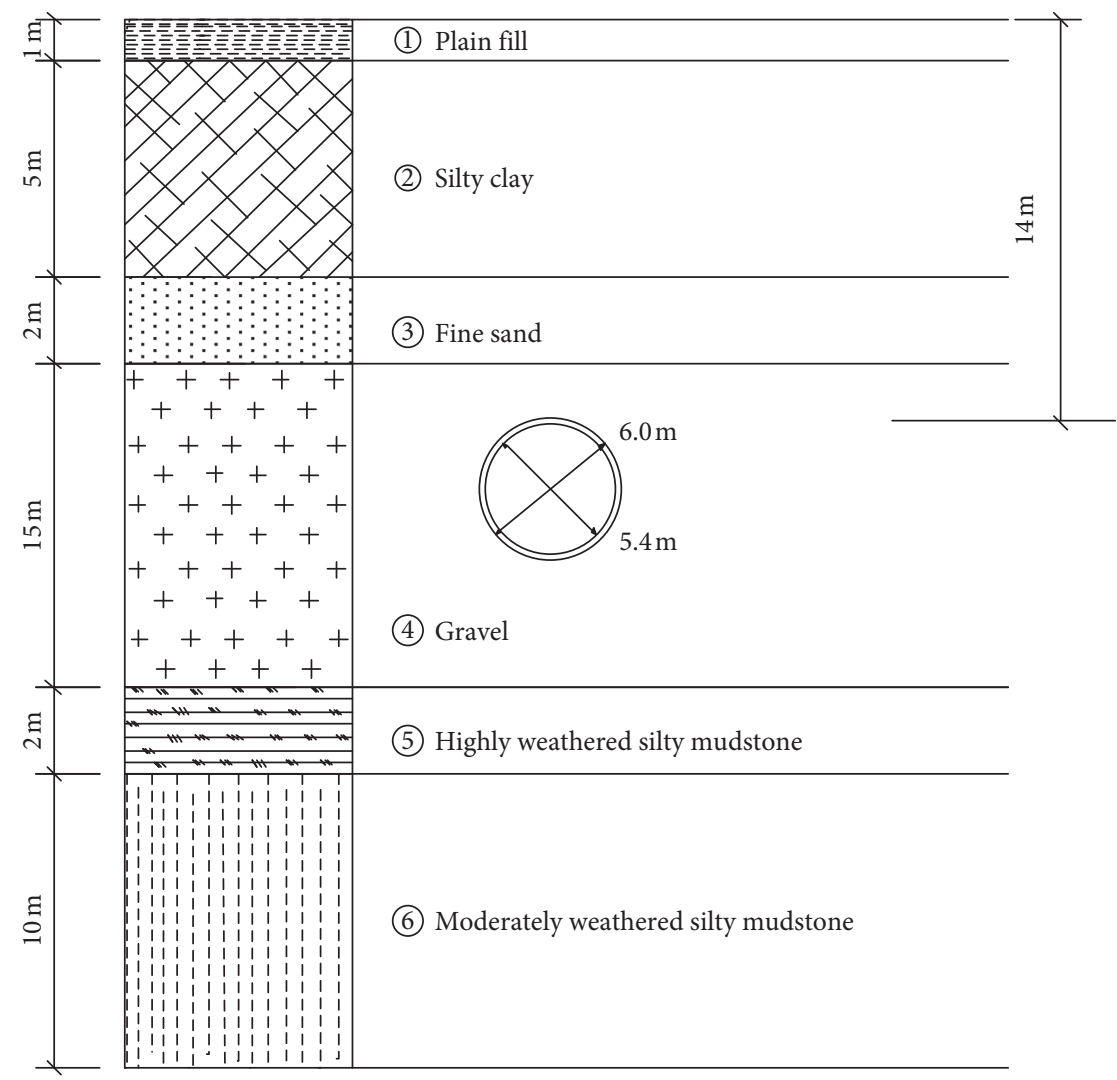

FIGURE 2: Profile of soil layers surrounding the shield tunnel.

TABLE 1: Excavation design for the concerned shield tunneling interval.

\begin{tabular}{llc}
\hline Step no. & Excavation time & Ring no. \\
\hline 1 & $2012 / 9 / 3015: 00$ & 836 \\
2 & $2012 / 10 / 107: 00$ & 838 \\
3 & $2012 / 10 / 1 \quad 15: 00$ & 839 \\
4 & $2012 / 10 / 207: 00$ & 840 \\
5 & $2012 / 10 / 215: 00$ & 841 \\
\hline
\end{tabular}

obtained from these two methods are in good agreement. It indicates the $4^{\text {th }}$ order HPCE-based surrogate models can well approximate the $3 \mathrm{D}$ numerical models and replace the deterministic finite difference analyses to accurately calculate the $u_{j}$ at each excavation step in this example.

3.4. Sequential Probabilistic Back Analysis Results. In this section, the BUS approach is employed to infer the posterior distributions of $E_{1}, E_{2}$, and $q$ via the sequential probabilistic back analysis using the time-series monitoring data of ground settlement as shown in Figure 3. Based on the tradeoff between the computational accuracy and efficiency, the number of samples at each subset level $N_{l}=5000$ and conditional probability $p_{0}=0.1$ are chosen. Following Miro et al. [21], the standard deviations of measurement errors $\sigma_{\varepsilon_{m_{j}}}=2.0 \mathrm{~mm}$ are used. Figures 6-8 compare the posterior PDFs of $E_{1}, E_{2}$, and $q$ estimated from the five different excavation steps, respectively. The prior PDFs of $E_{1}, E_{2}$, and

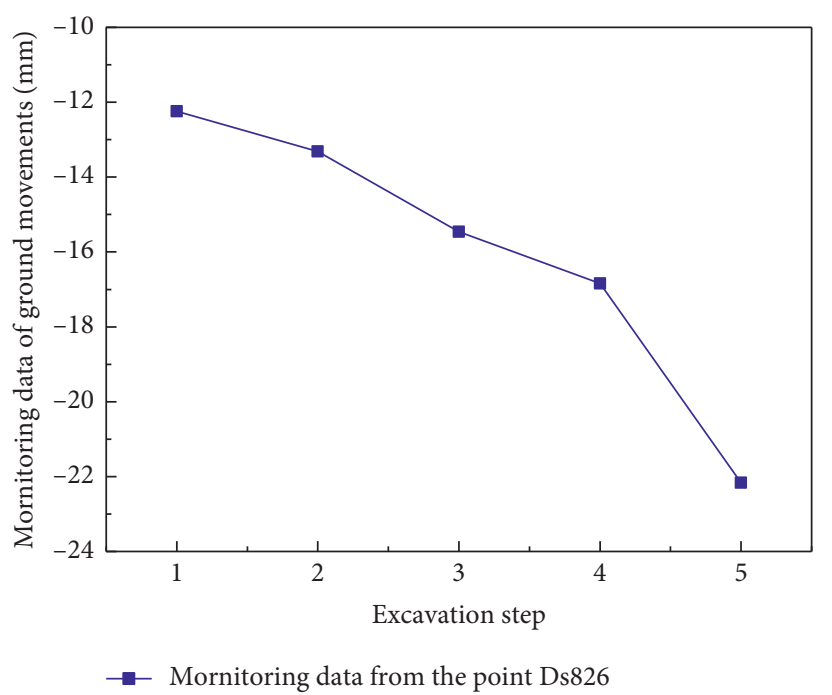

Figure 3: Time-series monitoring data of tunneling-induced ground settlement.

$q$ are also plotted in Figures 6-8, respectively, for comparison. As observed from Figures 6-8, the posterior PDF curves of $E_{1}, E_{2}$, and $q$ get steeper and narrower as the shield tunnel advances, and are much more peaked than the corresponding prior PDFs. The posterior means of $E_{1}$ and $E_{2}$ become smaller and smaller, while that of $q$ becomes larger and larger as the shield tunnel progresses. This is consistent 


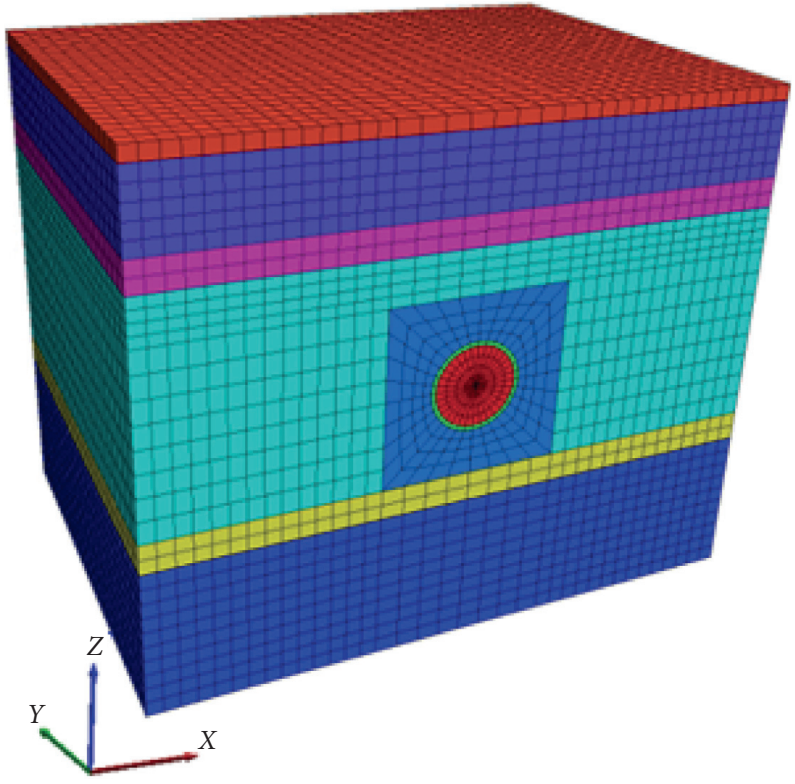

Figure 4: 3D finite difference model for the No. 1 Nanchang Metro Line tunnel.

TABLE 2: Geomechanical parameters for different soil layers.

\begin{tabular}{|c|c|c|c|c|c|}
\hline Soil layers & Density $\left(\mathrm{kg} / \mathrm{m}^{3}\right)$ & Young's modulus (MPa) & Poisson's ratio & Cohesion $(\mathrm{kPa})$ & Friction angle $\left({ }^{\circ}\right)$ \\
\hline Plain fill & 1813 & 15 & 0.42 & 5 & 10 \\
\hline Silty clay & 1933 & 15 & 0.35 & 44.7 & 19.3 \\
\hline Fine sand & 1913 & 16 & 0.4 & 1 & 30 \\
\hline Gravel & 1893 & 28 & 0.39 & 1 & 36 \\
\hline Highly weathered silty mudstone & 2050 & 120 & 0.3 & 60 & 37 \\
\hline Moderately weathered silty mudstone & 2390 & 450 & 0.39 & 120 & 32 \\
\hline
\end{tabular}

TABle 3: Prior statistics of three random variables.

\begin{tabular}{|c|c|c|c|c|}
\hline Random variable & Mean $(\mathrm{MPa})$ & Standard deviation $(\mathrm{MPa})$ & $\mathrm{COV}$ & Distribution \\
\hline Young's modulus of gravel layer, $E_{1}$ & 28 & 4.2 & 0.15 & Lognormal \\
\hline Young's modulus of silty clay layer, $E_{2}$ & 15 & 2.25 & 0.15 & Lognormal \\
\hline Uniform vehicle load, $q$ & 10 & 1.0 & 0.1 & Lognormal \\
\hline
\end{tabular}

with the post-event investigations and the common sense that the increase in the ground deformation is usually caused by the reduction of soil stuffiness or the increase of external loads. Significant changes can be observed on the posterior PDFs of $E_{1}, E_{2}$, and $q$ when the time-series monitoring data are sequentially incorporated in the probabilistic back analysis. This lies in the fact that the occurrence position of ground collapse is close to the $827^{\text {th }}$ ring and the ground settlement collected from the monitoring point Ds826 sharply increases at the $5^{\text {th }}$ excavation step (see Figure 3 ). It indicates the proposed approach not only can make full use of the time-series monitoring data to effectively update the statistics and reduce the uncertainties of geomechanical parameters, but also can well characterize the realistic change trends of surrounding subsoil properties.

Additionally, the COVs of $E_{1}, E_{2}$, and $q$ decrease successively from the prior COVs as the monitoring data are sequentially used in the probabilistic back analysis, as shown in Figure 9. The prior COVs of $E_{1}, E_{2}$, and $q$ are $0.15,0.15$, and 0.1 , respectively, which are reduced to 0.1 , 0.12 , and 0.085 at the $3^{\text {rd }}$ excavation step, and to $0.07,0.11$, and 0.08 at the $5^{\text {th }}$ excavation step. It is interesting to note that the uncertainty of $E_{1}$ is reduced the most, which implies the gravel layer affects the ground subsidence the most. The uncertainties of the geomechanical parameters associated with the shield tunnel have been significantly reduced through a Bayesian back analysis in a sequential manner.

3.5. Reliability Updating Results of Ground Settlements. Based on the obtained posterior distributions of the uncertain geomechanical parameters for each excavation step, the reliability of tunneling-induced ground settlements can be updated using equations (9) and (10). An admissible threshold of ground settlement $u_{\max }=30 \mathrm{~mm}$ is selected for 
TABLE 4: Expansion terms and coefficients of the $4^{\text {th }}$ order HPCE for the $1^{\text {st }}$ excavation step.

\begin{tabular}{|c|c|c|c|c|c|c|c|}
\hline No. & Term & Coefficient & Value & No. & Term & Coefficient & Value \\
\hline 1 & 1 & $a_{0}$ & 12.08 & 19 & $\xi_{2}^{2} \xi_{3}-\xi_{3}$ & $a_{18}$ & -0.03 \\
\hline 2 & $\xi_{1}$ & $a_{1}$ & -1.38 & 20 & $\xi_{1} \xi_{2} \xi_{3}$ & $a_{19}$ & -0.02 \\
\hline 3 & $\xi_{2}$ & $a_{2}$ & -0.32 & 21 & $\xi_{1}^{4}-6 \xi_{1}^{2}+3$ & $a_{20}$ & 0.01 \\
\hline 4 & $\xi_{3}$ & $a_{3}$ & 0.57 & 22 & $\xi_{2}^{4}-6 \xi_{2}^{2}+3$ & $a_{21}$ & -0.05 \\
\hline 5 & $\xi_{1}^{2}-1$ & $a_{4}$ & 0.27 & 23 & $\xi_{3}^{4}-6 \xi_{3}^{2}+3$ & $a_{22}$ & -0.01 \\
\hline 6 & $\xi_{2}^{2}-1$ & $a_{5}$ & -0.16 & 24 & $\xi_{1} \xi_{2}^{3}-\xi_{1} \xi_{2}$ & $a_{23}$ & 0.04 \\
\hline 7 & $\xi_{3}^{2}-1$ & $a_{6}$ & 0.01 & 25 & $\xi_{1} \xi_{3}^{3}-\xi_{1} \xi_{3}$ & $a_{24}$ & 0 \\
\hline 8 & $\xi_{1} \xi_{2}$ & $a_{7}$ & 0.02 & 26 & $\xi_{1}^{3} \xi_{2}-\xi_{1} \xi_{2}$ & $a_{25}$ & 0 \\
\hline 9 & $\xi_{1} \xi_{3}$ & $a_{8}$ & -0.15 & 27 & $\xi_{2} \xi_{3}^{2}-\xi_{2} \xi_{3}$ & $a_{26}$ & -0.03 \\
\hline 10 & $\xi_{2} \xi_{3}$ & $a_{9}$ & -0.01 & 28 & $\xi_{1}^{3} \xi_{3}-\xi_{1} \xi_{3}$ & $a_{27}$ & -0.04 \\
\hline 11 & $\xi_{1}^{3}-3 \xi_{1}$ & $a_{10}$ & -0.10 & 29 & $\xi_{2}^{3} \xi_{3}-\xi_{2} \xi_{3}$ & $a_{28}$ & 0.04 \\
\hline 12 & $\xi_{2}^{3}-3 \xi_{2}$ & $a_{11}$ & -0.01 & 30 & $\xi_{1}^{2} \xi_{2}^{2}-\xi_{1}^{2}-\xi_{2}^{2}+1$ & $a_{29}$ & -0.01 \\
\hline 13 & $\xi_{3}^{3}-3 \xi_{3}$ & $a_{12}$ & 0 & 31 & $\xi_{1}^{2} \xi_{3}^{2}-\xi_{1}^{2}-\xi_{3}^{2}+1$ & $a_{30}$ & 0.02 \\
\hline 14 & $\xi_{1} \xi_{2}^{2}-\xi_{1}$ & $a_{13}$ & -0.01 & 32 & $\xi_{2}^{2} \xi_{3}^{2}-\xi_{2}^{2}-\xi_{3}^{2}+1$ & $a_{31}$ & 0.04 \\
\hline 15 & $\xi_{1} \xi_{3}^{2}-\xi_{1}$ & $a_{14}$ & -0.01 & 33 & $\xi_{1}^{2} \xi_{2} \xi_{3}-\xi_{2} \xi_{3}$ & $a_{32}$ & 0.07 \\
\hline 16 & $\xi_{1}^{2} \xi_{2}-\xi_{2}$ & $a_{15}$ & 0.06 & 34 & $\xi_{1} \xi_{2}^{2} \xi_{3}-\xi_{1} \xi_{3}$ & $a_{33}$ & -0.03 \\
\hline 17 & $\xi_{2} \xi_{3}^{2}-\xi_{2}$ & $a_{16}$ & 0.03 & 35 & $\xi_{1} \xi_{2} \xi_{3}^{2}-\xi_{1} \xi_{2}$ & $a_{34}$ & -0.03 \\
\hline 18 & $\xi_{1}^{2} \xi_{3}-\xi_{3}$ & $a_{17}$ & 0.09 & & & & \\
\hline
\end{tabular}

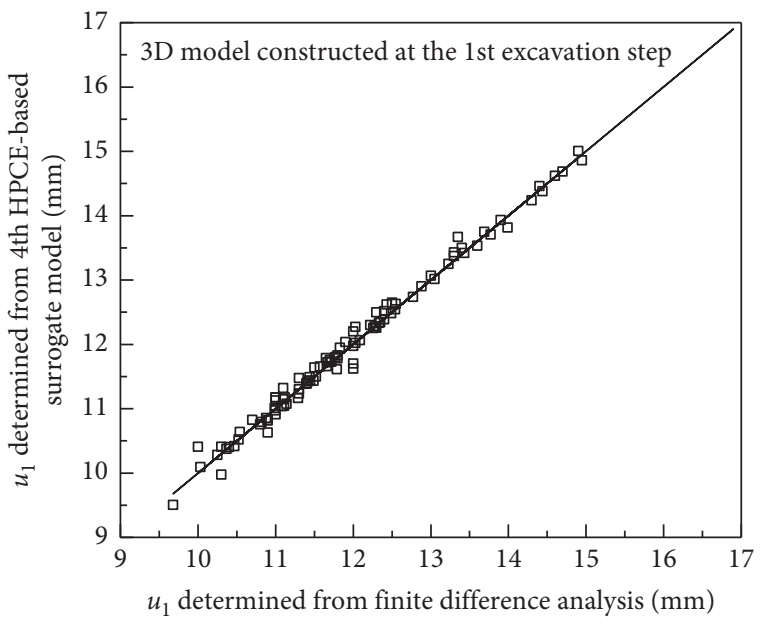

(a)

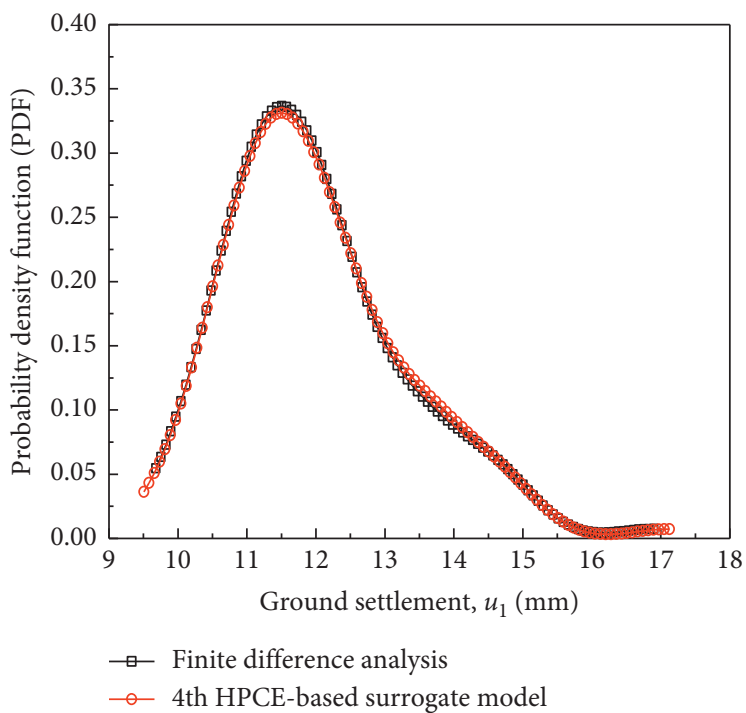

(b)

Figure 5: Continued. 


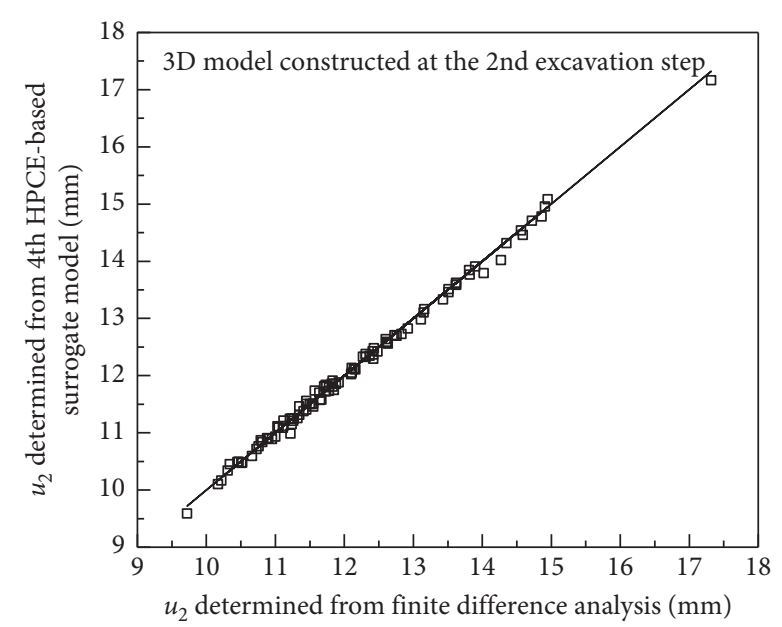

(c)

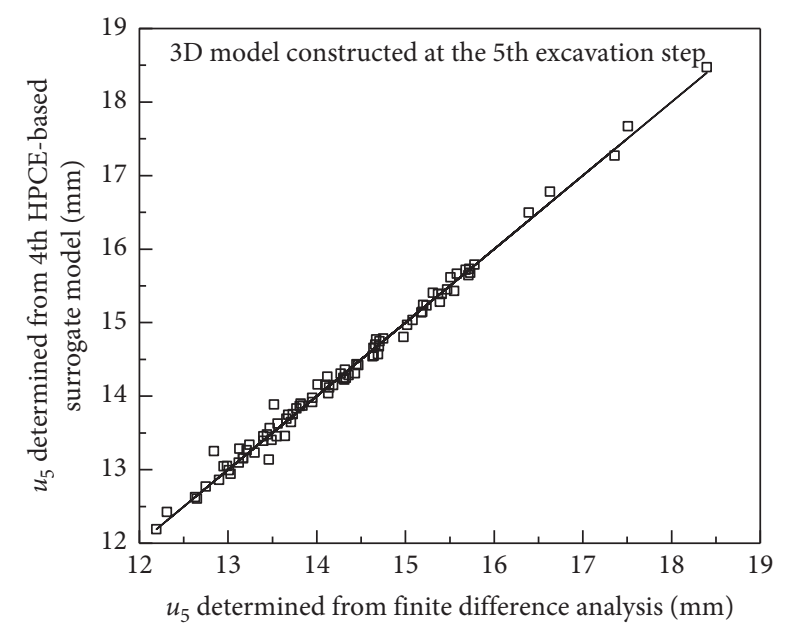

(e)

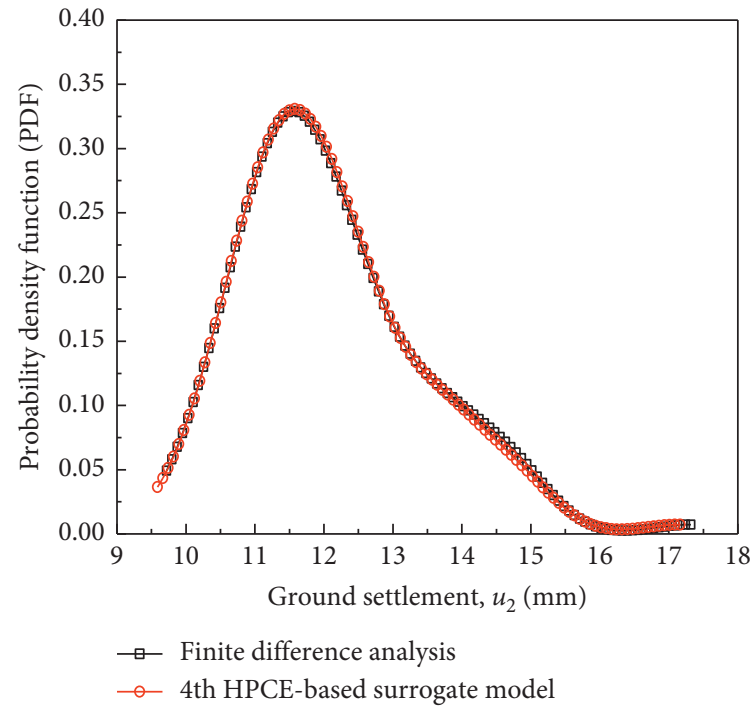

(d)

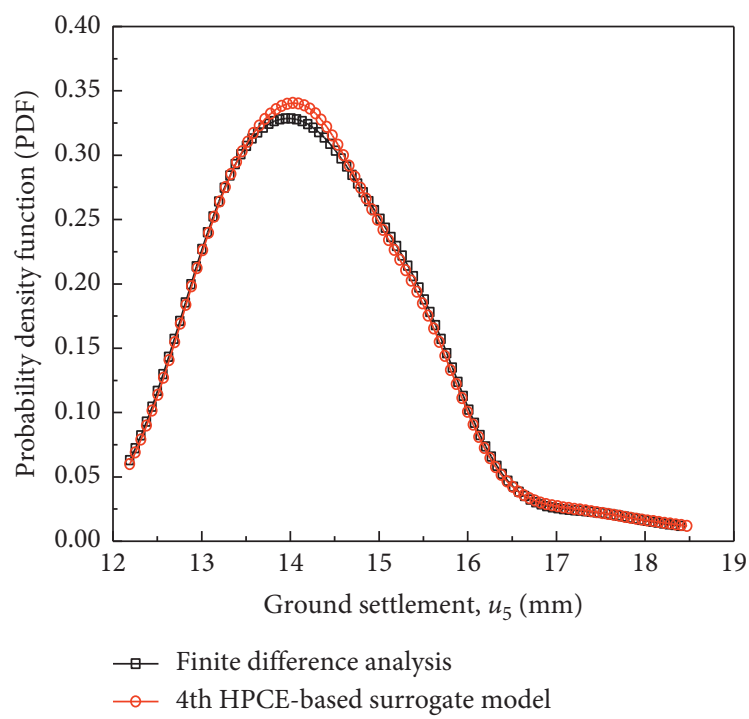

(f)

Figure 5: Validation of the surrogate models underlying three representative excavation steps. (a) Comparison of $u_{1}$. (b) Comparison of the PDF of $u_{1}$. (c) Comparison of $u_{2}$. (d) Comparison of the PDF of $u_{2}$. (e) Comparison of $u_{5}$. (f) Comparison of the PDF of $u_{5}$.

illustration. Figure 10 presents the variation of the posterior probability of ground collapse with the excavation step. As seen from Figure 10, the posterior probability of ground collapse increases continuously as the tunnel starts to advance. Then it increases dramatically at the $2^{\text {nd }}$ excavation step and exceeds the prior probability of ground collapse $\left(5.152 \times 10^{-5}\right)$ and increases furthermore at the $3^{\text {rd }}$ excavation step until reaching $0.36 \%$ at the $5^{\text {th }}$ excavation step. The variation trend of the posterior probability indicates a safety check and necessary support measures shall be timely taken at the $3^{\text {rd }}$ excavation step to control the monotonous increase of ground settlement. Otherwise, the occurrence probability of ground collapse due to the shield tunneling will eventually be large and unacceptable. Moreover, the variation trend of the posterior probability with the time is consistent with that of the time-series monitoring data as shown in Figure 3.

For the case of the $5^{\text {th }}$ excavation step, the posterior probability of ground collapse $\left(P_{f, 5}\right)$ estimated from the proposed approach is $0.36 \%$. To calculate such a probability, the proposed approach needs performing $5 \times 70$ runs of $3 \mathrm{D}$ deterministic finite difference analyses of the tunnelinginduced ground settlements to construct five surrogate models and additional probabilistic back analysis and reliability updating. For the same problem, the direct MCS requires more than 27677 runs of 3D deterministic finite difference analyses for achieving a target $\operatorname{COV}_{P_{f 5}}$ below $10 \%$. This is because the least number of samples 


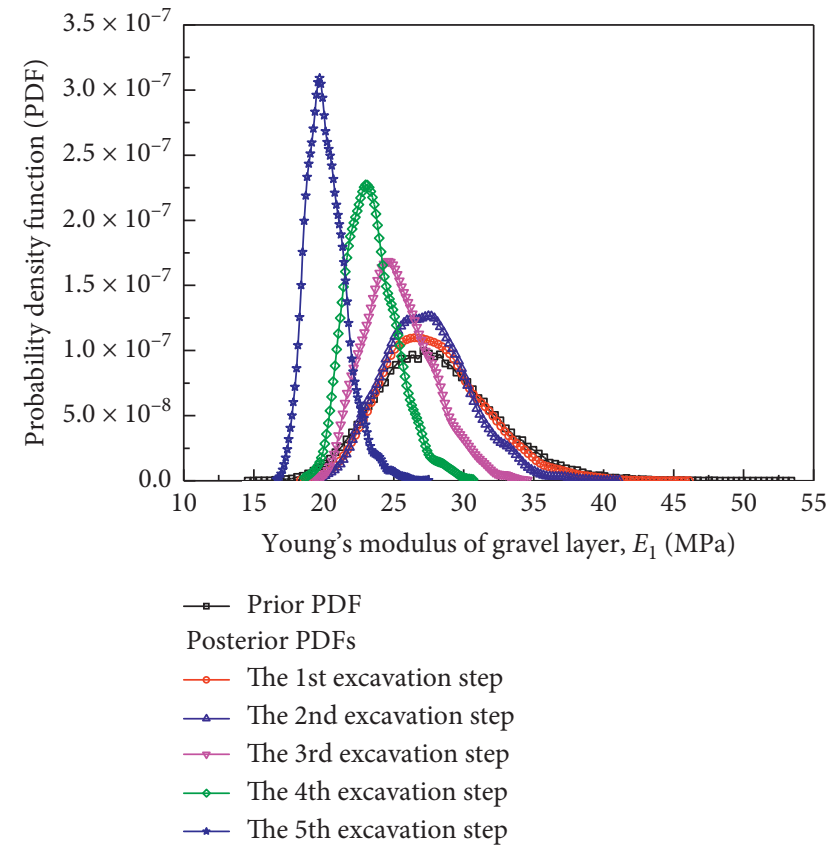

Figure 6: Comparison of the posterior PDFs of Young's modulus of gravel layer for different excavation steps.

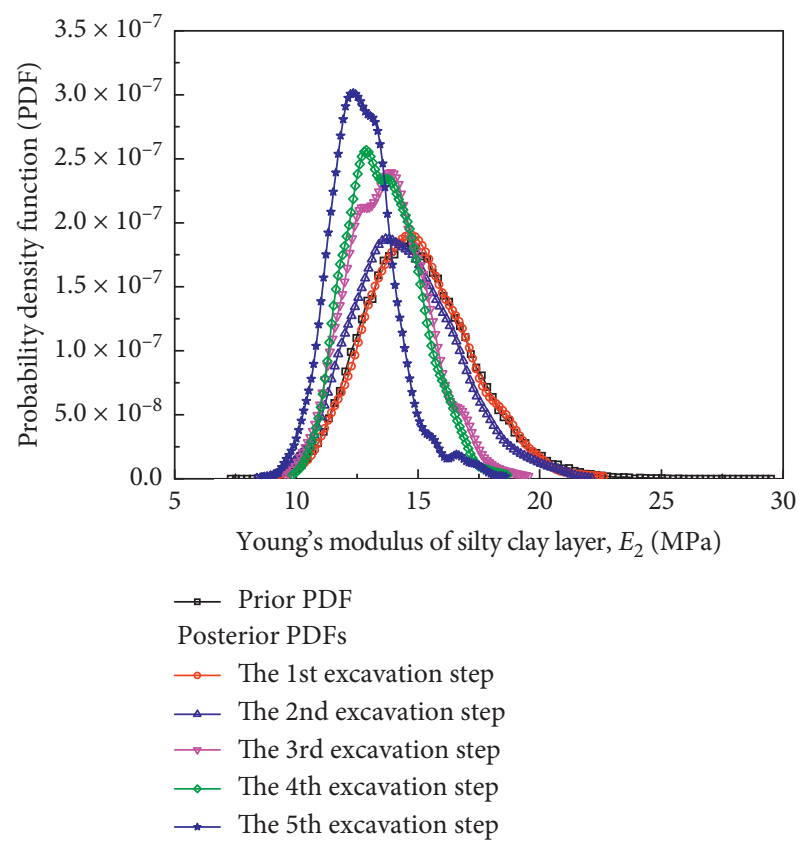

FIgURe 7: Comparison of the posterior PDFs of Young's modulus of silty clay layer for different excavation steps.

required for the MCS to estimate $P_{f, 5}$ is calculated by $N_{\text {sim }} \geq\left(1-P_{f, 5}\right) /\left(P_{f, 5}\left(\operatorname{COV}_{P_{f, 5}}\right)^{2}\right)[22]$. The computational time required for one run of $3 \mathrm{D}$ deterministic finite difference analysis is 800 seconds when the computations are

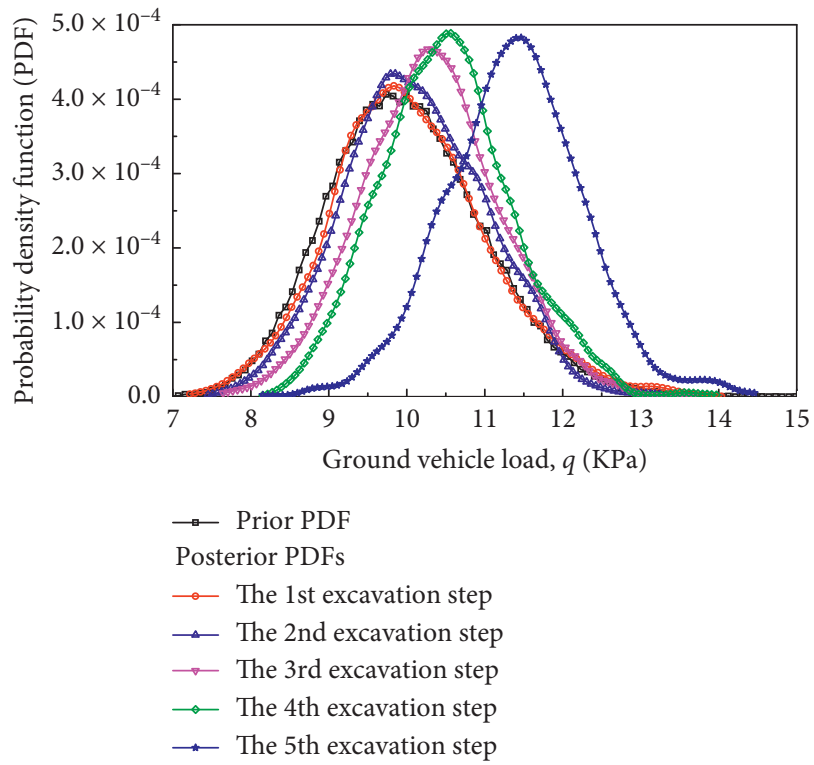

FIGURE 8: Comparison of the posterior PDFs of ground vehicle load for different excavation steps.

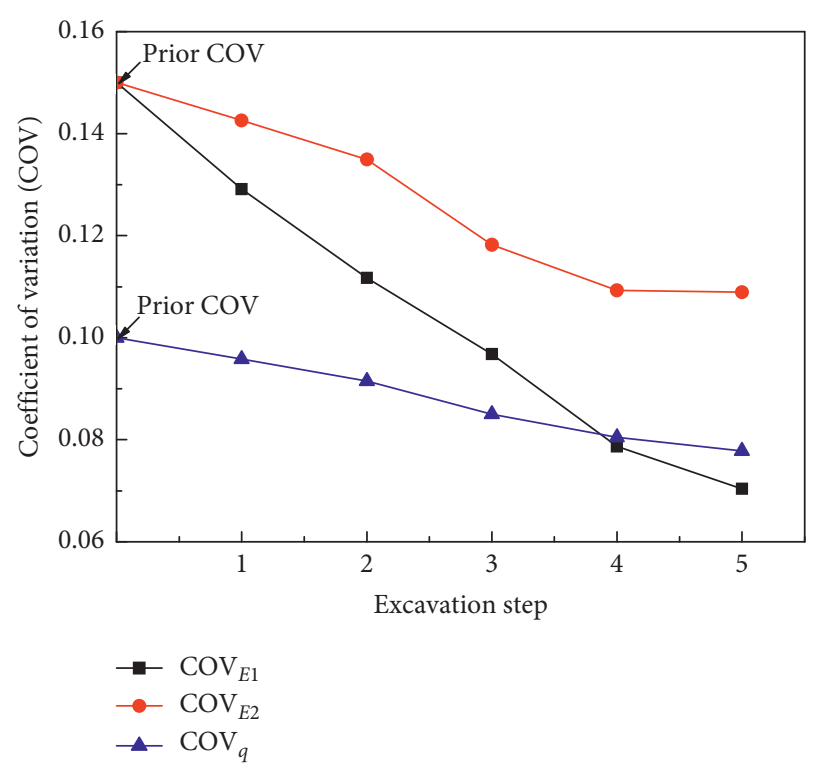

FIGURE 9: Variation of the coefficients of variation of input parameters with the excavation step.

performed on a desktop with 8 GB RAM and one Intel Core i7-4790 CPU clocked at $3.6 \mathrm{GHz}$. The computational time taken on the probabilistic back analysis and reliability updating with the constructed surrogate models equals 18 seconds, which is only $1 / 44$ of that required for one run of 3D deterministic finite difference analysis. Based on these, about 6150 hours will be required for the direct MCS while $5 \times 800+18$ seconds (1.1 hours) are required for the proposed approach to calculate the posterior probability of 


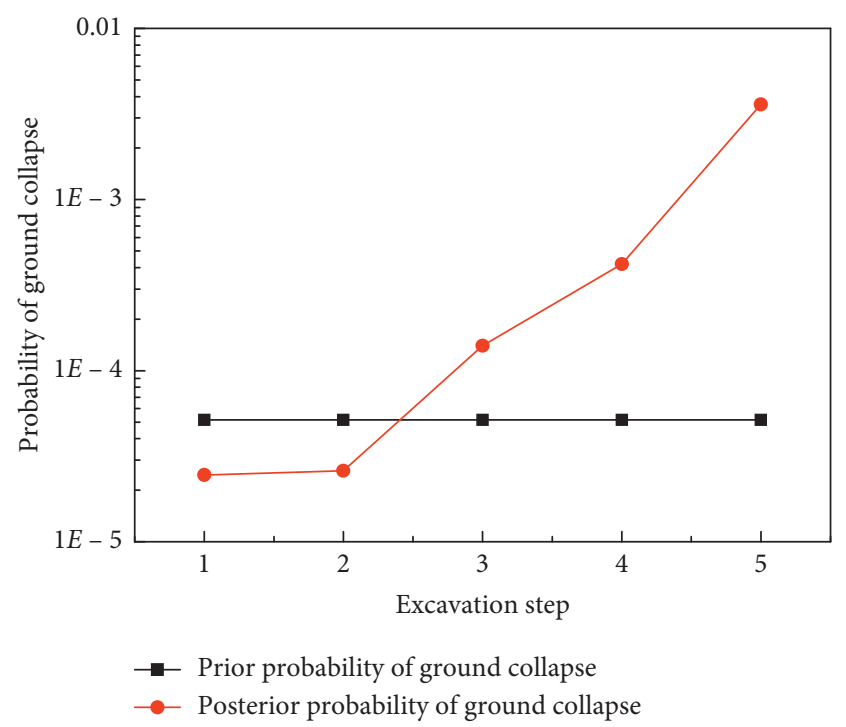

FIgURE 10: Comparison of prior and posterior probabilities of ground collapse induced by shield tunneling.

ground collapse at the $5^{\text {th }}$ excavation step. This confirms that the proposed approach is much more efficient in the probabilistic back analysis of the uncertain geomechanical parameters and the reliability updating. Such high efficiency will greatly facilitate the applications of the proposed approach in geotechnical engineering.

\section{Conclusions}

A BUS-based sequential probabilistic back analysis is proposed to estimate the uncertain geomechanical parameters and update the reliability of tunneling-induced ground settlements. The shield tunnel project of No. 1 Nanchang Metro Line in China is investigated to assess the effectiveness of the proposed approach. Several conclusions can be drawn from this study:

(1) The proposed approach can well infer the posterior distributions of uncertain geomechanical parameters by fully utilizing the time-series monitoring data. The reliability of tunneling-induced ground settlements is updated in a real-time manner. The computational efficiency has been improved through transforming the Bayesian back analysis problem into an equivalent structural reliability problem and constructing the surrogate models of the output responses of shield tunnels by the Hermite polynomial chaos expansion.

(2) By employing the proposed approach, the variation trends of the means of uncertain geomechanical parameters and the posterior probability of ground collapse match well with those of time-series monitoring data and the post-event investigations. The probability distributions of geomechanical parameters gradually converge to the target distribution, and the uncertainties of geomechanical parameters are reduced successively after updating. These demonstrate the effectiveness of the proposed approach.
(3) The sequential probabilistic back analysis and reliability updating results can provide an important reference for the reduction of the uncertainties of geomechanical parameters during shield tunnel excavation and, consequently, the mitigation of the potential risk of ground collapse. For the considered real example, the probability of ground collapse increases markedly from October 1, 2012, 7:00 to October 1, 2012, 15:00, which can provide valuable information for the practitioners to formulate early warning measures to prevent the occurrence of ground collapse accident.

\section{Data Availability}

Some or all data, models, or code generated or used during this study are available to the readers upon request. The items are listed as follows:

(1) Time-series monitoring data of tunneling-induced ground settlement

(2) Hermite polynomial chaos expansion code that is used for constructing the surrogate models of the output responses of shield tunnels

(3) BUS code that is used for inferring the posterior distribution of geomechanical parameters and estimating the posterior probability of ground collapse

\section{Conflicts of Interest}

The authors declare that there are no conflicts of interest regarding the publication of this paper.

\section{Acknowledgments}

This work was supported by the National Natural Science Foundation of China (Project nos. 41867036 and 41972280), Jiangxi Provincial Natural Science Foundation (Project nos. 2018ACB21017, 20181ACB20008 and 20192BBG70078), and Open Research Fund of State Key Laboratory of Geomechanics and Geotechnical Engineering (Project no. Z019019). The financial support is gratefully acknowledged.

\section{References}

[1] C. Camós, O. Špačková, D. Straub, and C. Molins, "Probabilistic approach to assessing and monitoring settlements caused by tunneling," Tunnelling and Underground Space Technology, vol. 51, pp. 313-325, 2016.

[2] V. H. Franco, G. D. F. N. Gitirana, and A. P. De Assis, "Probabilistic assessment of tunneling-induced building damage," Computers and Geotechnics, vol. 113, Article ID 103097, 2019.

[3] G. Mollon, D. Dias, and A.-H. Soubra, "Probabilistic analyses of tunneling-induced ground movements," Acta Geotechnica, vol. 8, no. 2, pp. 181-199, 2013.

[4] W. Gao and M. Ge, "Back analysis of rock mass parameters and initial stress for the longtan tunnel in China," Engineering with Computers, vol. 32, no. 3, pp. 497-515, 2016.

[5] W. Gong, C. H. Juang, J. R. Martin, H. Tang, Q. Wang, and H. Huang, "Probabilistic analysis of tunnel longitudinal 
performance based upon conditional random field simulation of soil properties," Tunnelling and Underground Space Technology, vol. 73, pp. 1-14, 2018.

[6] J. Hu, W. Liu, Y. Pan, and H. Zeng, "Site measurement and study of vertical freezing wall temperatures of a large-diameter shield tunnel," Advances in Civil Engineering, vol. 2019, Article ID 8231458, 11 pages, 2019.

[7] S.-Y. Chi, J.-C. Chern, and C.-C. Lin, "Optimized backanalysis for tunneling-induced ground movement using equivalent ground loss model," Tunnelling and Underground Space Technology, vol. 16, no. 3, pp. 159-165, 2001.

[8] C. Zhu, H. Zhao, and M. Zhao, "Back analysis of geomechanical parameters in underground engineering using artificial bee colony," The Scientific World Journal, vol. 2014, Article ID 693812, 13 pages, 2014.

[9] Y. Sun, J. Huang, W. Jin, S. W. Sloan, and Q. Jiang, "Bayesian updating for progressive excavation of high rock slopes using multi-type monitoring data," Engineering Geology, vol. 252, pp. 1-13, 2019.

[10] K.-K. Phoon and F. H. Kulhawy, "Characterization of geotechnical variability," Canadian Geotechnical Journal, vol. 36, no. 4, pp. 612-624, 1999.

[11] D.-Q. Li, S.-H. Jiang, Y.-F. Chen, and C.-B. Zhou, "Reliability analysis of serviceability performance for an underground cavern using a non-intrusive stochastic method," Environmental Earth Sciences, vol. 71, no. 3, pp. 1169-1182, 2014.

[12] X. M. Li, "Study on ground subsidence induced by earth pressure balanced shield tunneling," PhD Thesis, Nanjing University, Nanjing, China, 2014.

[13] H. Huang, W. Gong, S. Khoshnevisan, C. H. Juang, D. Zhang, and L. Wang, "Simplified procedure for finite element analysis of the longitudinal performance of shield tunnels considering spatial soil variability in longitudinal direction," Computers and Geotechnics, vol. 64, pp. 132-145, 2015.

[14] S.-H. Jiang and J.-S. Huang, "Efficient slope reliability analysis at low-probability levels in spatially variable soils," Computers and Geotechnics, vol. 75, pp. 18-27, 2016.

[15] S.-H. Jiang, J. Huang, C. Yao, and J. Yang, "Quantitative risk assessment of slope failure in 2-D spatially variable soils by limit equilibrium method," Applied Mathematical Modelling, vol. 47, pp. 710-725, 2017.

[16] H. Cheng, J. Chen, R. Chen, J. Huang, and J. Li, “Three-dimensional analysis of tunnel face stability in spatially variable soils," Computers and Geotechnics, vol. 111, pp. 76-88, 2019.

[17] C. Haas and H. H. Einstein, "Updating the decision aids for tunneling," Journal of Construction Engineering and Management, vol. 128, no. 1, pp. 40-48, 2002.

[18] O. Špačková and D. Straub, "Probabilistic assessment of tunnel construction performance based on data," Tunnelling and Underground Space Technology, vol. 37, pp. 62-78, 2013.

[19] D. Park and E.-S. Park, "Inverse parameter fitting of tunnels using a response surface approach," International Journal of Rock Mechanics and Mining Sciences, vol. 77, pp. 11-18, 2015.

[20] W. Liu, X. Luo, J. Huang, L. Hu, and M. Fu, "Probabilistic analysis of tunnel face stability below river using Bayesian framework," Mathematical Problems in Engineering, vol. 2018, Article ID 1450683, 8 pages, 2018.

[21] S. Miro, M. König, D. Hartmann, and T. Schanz, "A probabilistic analysis of subsoil parameters uncertainty impacts on tunnel-induced ground movements with a back-analysis study," Computers and Geotechnics, vol. 68, pp. 38-53, 2015.

[22] H. S. Ang and W. H. Tang, Probability Concepts in Engineering: Emphasis on Applications to Civil and Environmental
Engineering, John Wiley \& Sons, New York City, NY, USA, 2 edition, 2007.

[23] D. Straub and I. Papaioannou, "Bayesian updating with structural reliability methods," Journal of Engineering $\mathrm{Me}$ chanics, vol. 141, no. 3, Article ID 04014134, 2015.

[24] W. G. Zhang and A. T. C. Goh, "Multivariate adaptive regression splines for analysis of geotechnical engineering systems," Computers and Geotechnics, vol. 48, pp. 82-95, 2013.

[25] D.-Q. Li, D. Zheng, Z.-J. Cao, X.-S. Tang, and K.-K. Phoon, "Response surface methods for slope reliability analysis: review and comparison," Engineering Geology, vol. 203, pp. 314, 2016.

[26] W. Zhang and A. T. C. Goh, "Multivariate adaptive regression splines and neural network models for prediction of pile drivability," Geoscience Frontiers, vol. 7, no. 1, pp. 45-52, 2016.

[27] X. Liu, D.-Q. Li, Z.-J. Cao, and Y. Wang, "Adaptive monte carlo simulation method for system reliability analysis of slope stability based on limit equilibrium methods," Engineering Geology, vol. 264, Article ID 105384, 2020.

[28] G. Mollon, D. Dias, and A.-H. Soubra, "probabilistic analysis of circular tunnels in homogeneous soil using response surface methodology," Journal of Geotechnical and Geoenvironmental Engineering, vol. 135, no. 9, pp. 1314-1325, 2009.

[29] D. Li, Y. Chen, W. Lu, and C. Zhou, "Stochastic response surface method for reliability analysis of rock slopes involving correlated non-normal variables," Computers and Geotechnics, vol. 38, no. 1, pp. 58-68, 2011.

[30] R. G. Ghanem and P. D. Spanos, Stochastic Finite Element: A Spectral Approach-Revised Version, Dover Publication, Mineola, NY, USA, 2003.

[31] S. K. Choi, R. A. Canfield, and R. V. Grandhi, "Estimation of structural reliability for gaussian random fields," Structure and Infrastructure Engineering, vol. 2, no. 3-4, pp. 161-173, 2006.

[32] I. Papaioannou and D. Straub, "Reliability updating in geotechnical engineering including spatial variability of soil," Computers and Geotechnics, vol. 42, pp. 44-51, 2012.

[33] S.-K. Au and J. L. Beck, "Estimation of small failure probabilities in high dimensions by subset simulation," Probabilistic Engineering Mechanics, vol. 16, no. 4, pp. 263-277, 2001.

[34] J. Huang, G. Fenton, D. V. Griffiths, D. Li, and C. Zhou, "On the efficient estimation of small failure probability in slopes," Landslides, vol. 14, no. 2, pp. 491-498, 2017.

[35] W. Betz, I. Papaioannou, J. L. Beck, and D. Straub, "Bayesian inference with subset simulation: strategies and improvements," Computer Methods in Applied Mechanics and Engineering, vol. 331, pp. 72-93, 2018.

[36] S.-H. Jiang, I. Papaioannou, and D. Straub, "Bayesian updating of slope reliability in spatially variable soils with insitu measurements," Engineering Geology, vol. 239, pp. 310320,2018

[37] S.-H. Jiang, J. Huang, X.-H. Qi, and C.-B. Zhou, "Efficient probabilistic back analysis of spatially varying soil parameters for slope reliability assessment," Engineering Geology, vol. 271, Article ID 105597, 2020.

[38] D. Yang, H. Huang, and J. Zhang, "Study on probability distribution of vehicle load and its load effect," China Journal of Guangzhou University, vol. 13, no. 5, pp. 56-60, 2014.

[39] Jiangxi Survey and Design Institute, Geotechnical Investigation, Nanchang Metro Line, Nanchang, China, 2009.

[40] J. Bauer and W. Puła, "Reliability with respect to settlement limit-states of shallow foundations on linearly-deformable 
subsoil," Computers and Geotechnics, vol. 26, no. 3-4, pp. 281-308, 2000.

[41] G. B. Baecher and J. T. Christian, Reliability and Statistics in Geotechnical Engineering, John Wiley \& Sons, New York City, NY, USA, 2003.

[42] Y. Li, L. Tang, Z. Liu, and Y. Liu, "Statistics and probability analysis of vehicle overloads on a rigid frame bridge from long-term monitored strains," Smart Structures and Systems, vol. 9, no. 3, pp. 287-301, 2012. 